\title{
La construcción de la agenda de los cibermedios. Estudio comparativo con las preferencias temáticas de lectores y usuarios de Twitter
}

\section{The agenda-building process on digital news media. A comparative study with issue preferences of readers and Twitter users}

Pedro-Luis Pérez-Díaz. Universidad Católica San Antonio de Murcia. España.

plperez@ucam.edu

$[\underline{\mathrm{CV}}]$ (2)

Enrique Arroyas Langa. Universidad Católica San Antonio de Murcia. España. earroyas@ucam.edu

$[\mathrm{CV}]$ (D)

Rocío Zamora Medina. Universidad de Murcia. España.

rzamoramedina@um.es

$[\mathrm{CV}]$ (1) G

Agradecimientos: Fundación Séneca, Agencia de Ciencia y Tecnología de la Región de Murcia.

Cómo citar este artículo / Referencia normalizada

Pérez-Díaz, P. L., Arroyas Langa, E. y Zamora Medina, R. (2020). La construcción de la agenda de los cibermedios. Estudio comparativo con las preferencias temáticas de lectores y usuarios de Twitter. Revista Latina de Comunicación Social, 75, 225-244.

https://www.doi.org/10.4185/RLCS-2020-1424

\section{RESUMEN}

Introducción: Esta investigación tiene como propósito analizar la actividad de la audiencia en la construcción de la agenda periodística de cuatro cibermedios generalistas líderes en el ámbito español (El País, El Mundo, El Confidencial y El Diario). Metodología: A partir de un análisis de contenido ( $n=3.600$ temas recogidos durante 15 días de junio de 2017), se registra la coincidencia entre los temas de portada, las clasificaciones de 'lo más leído' y los 'temas del momento' asociados en Twitter, entre otras variables. Resultados y conclusiones: Se concluye que los cibermedios estudiados presentan una agenda de portada sensible a la participación ciudadana, que se traduce en un protagonismo más duradero para aquellos asuntos que coinciden con los más leídos o los trending topics. Aunque prevalece el distanciamiento entre los tratamientos informativos preferidos por los periodistas (duros) y los más leídos (blandos), son los primeros los que logran más participación, en concomitancia con otros factores como la coincidencia interagenda, la permanencia y la posición en portada.

PALABRAS CLAVE: agenda informativa; cibermedios; periodismo digital; participación ciudadana; Twitter; analíticas web. 


\begin{abstract}
Introduction: The purpose of this research is to analyze the audience activity in a more participatory process of agenda-building on four leading digital news media in Spain (El País, El Mundo, El Confidencial and El Diario). Methodology: Based on a content analysis ( $n=3,600$ topics collected during 15 days of June 2017), we register the coincidence between topics from homepages, the 'most read' classifications and the trending topics associated on Twitter. Results and conclusions: It is concluded that the studied digital news media exhibit an agenda sensitive to citizen participation, which translates into a more lasting prominence for those issues that coincide with the most read stories or trending topics. Although the distance between the news treatments favored by journalists (hard) and the most read stories (soft) prevails, it is the former ones that manage to catalyze greater participatory metrics, in concomitance with other factors such as the interagenda coincidence, the permanence and position on the homepage or the publishing time.
\end{abstract}

KEYWORDS: news agenda; digital news media; digital journalism; citizen participation; Twitter; web analytics.

\title{
CONTENIDO
}

1. Introducción. 1.1. Un gatekeeping participativo basado en la actividad de la audiencia. 1.2. La brecha en el tratamiento informativo preferido por periodistas y ciudadanos. 1.3. La conversación en redes sociales en el proceso de agenda-building. 2. Metodología. 2.1. Población y muestra. 2.2. Instrumentos de recogida de información y procedimiento. 3. Resultados. 3.1. Selección informativa. 3.2. Coincidencia interagenda.3.3. Evolución de los temas. 3.4. Tratamiento informativo de los temas. 3.5. Métricas participativas asociadas a los temas. 4. Discusión y conclusiones. 5. Referencias.

Traducción del resumen realizada por Miriam Salar López. Traductora profesional (York, Inglaterra).

\section{Introducción}

Los flujos periodísticos de decisión que conducen a la selección informativa que tradicionalmente han surgido de un juicio profesional aislado mutan en el contexto digital, en virtud de una cultura participativa que celebra la visibilización de los intereses de la audiencia mediante el contenido generado por el usuario y el feedback diario en la construcción de la agenda mediática (Tandoc Jr., 2014). La literatura sobre el periodismo participativo persiste en constatar que los profesionales no comparten con sus lectores aspectos sensibles de su labor que se relacionan con su autoridad ocupacional (Abbott, 2017), como el gatekeeping. Con todo, cada vez son más los trabajos que describen cómo esta fase de la producción informativa digital podría estar condicionada por las dinámicas y actividades de la ciudadanía conectada, lo que vendría a reivindicar la vieja idea de que "la verdadera materia del periodismo es la conversación que el público está manteniendo consigo mismo" (Rosen, 1997, p. 191).

A mediados de los años noventa del pasado siglo, la arquitectura distribuida de Internet, en contraposición a las estructuras de concentración de los mass media, ya planteaban un reto: la dispersión de la información en nodos de datos y la interactividad pondrían en peligro el control de la agenda informativa (Newhagen y Levy, 1996). Con la llegada de una web mucho más social, los lectores de los cibermedios terminarían por encontrar ante sí toda una serie de interactividades que repercuten en la visibilidad de las noticias, como acceder a ellas, compartirlas, escribir comentarios o votar y puntuar su interés o calidad: "Los resultados de estas actividades se conocen colectivamente como la popularidad de noticia, una evaluación del valor de una noticia de acuerdo con la audiencia" (Shoemaker, Johnson, Seo y Wang, 2010, p. 57). 


\subsection{Un gatekeeping participativo basado en la actividad de la audiencia}

Así, Nielsen (2017) distingue un gatekeeping basado en la audiencia, en la que esta se embarca en un filtrado que, con mayor o menor consciencia y de forma individual o colaborativa, resulta en un contenido con cierta visibilidad generada por el usuario, como la denomina Singer (2014). La visibilidad derivada de estos comportamientos por parte de una audiencia activa (Singer et al., 2011) ha provocado un cambio de paradigma hacia un gatekeeping y un framing en red (Meraz y Papacharissi, 2016), en el que los ciudadanos conectados alteran la prominencia tradicional de determinados actores y promocionan nuevos gatekeepers, impulsan issues y modelan la interpretación misma de esos temas interpelando directamente a los profesionales y manifestando dinámicas de poder (Pérez-Díaz, Berná Sicilia y Arroyas Langa, 2016).

En un entorno digital poblado por audiencias de atención volátil, métricas y analíticas web han comenzado a modelar decisiones editoriales (Nguyen, 2013). Estos datos son precisamente usados en técnicas de selección informativa basadas en la agregación, en la que se emplea un software compuesto por algoritmos que recopilan contenidos relacionados con un determinado tópico o contexto, generalmente a partir de metadatos, votos o palabras clave. El ejemplo más evidente en cibermedios son los ubicuos apartados consagrados a 'lo más leído', 'lo más comentado' o 'lo más compartido', que visibilizan piezas a partir de parámetros puramente cuantitativos.

Como consecuencia, el eje consumidor pasivo/productor activo en el que tradicionalmente se trataba de encuadrar a la audiencia se ha visto afectado por formas participativas ligeras o de baja intensidad en forma de feedback, que condicionan y afectan al proceso de producción informativa, y más específicamente, a la configuración de la agenda política y mediática (Masip, Guallar, Suau y RuizCaballero, 2015; Lawrence, Radcliffe y Schmidt, 2017), dado que la visibilidad, prominencia o ubicación en portada de una noticia está poderosamente relacionada con el número de lecturas que ha recibido (Lee, Lewis y Powers, 2012).

\subsection{La brecha en el tratamiento informativo preferido por periodistas y ciudadanos}

A juicio de Manosevitch y Tenenboim (2017, p. 15), la jerarquización de contenidos obtenida mediante los mecanismos de agregación que impulsan apartados del tipo 'lo más leído, compartido o comentado' atrae tráfico web, proporciona un input a las decisiones editoriales y permite evaluar qué asuntos suscitan la atención pública. No obstante, el uso de estas analíticas para la confección de piezas informativas comporta toda una serie de peligros larvados (Tandoc Jr. y Thomas, 2015). La disonancia entre lo que es importante para los lectores - a menudo soft news - y los asuntos que preocupan a los periodistas descubre una brecha evidente que debe ser salvada (Boczkowski y Peer, 2011) para encontrar el equilibrio entre el interés público y el interés del público.

Asimismo, estos espacios en ocasiones se convierten en un escaparate de asuntos triviales e infoentretenimiento (Boczkowski y Mitchelstein, 2015; Morera Hernández, 2016; Welbers, van Atteveldt, Kleinnijenhuis, Ruigrok y Schaper, 2016), que pueden minar la seriedad y el prestigio del cibermedio. Esta discordancia temática provoca una brecha entre la oferta y la demanda de información de actualidad que Boczkowski y Mitchelstein (2015) denominan "brecha informativa" o news gap: "Mientras editores y periodistas tienden a dar prioridad a las noticias sobre asuntos públicos [...] los consumidores, en cambio, prefieren noticias sobre asuntos no públicos, como deportes, espectáculos, [temas] policiales y el clima" (Mitchelstein, Boczkowski, Wagner y Leiva, 2016, p. 1030). 
Otro peligro latente es que el medio acabe esclavizado ante los apetitos de la audiencia; una forma de proceder característica del modelo de negocio sensacionalista en el que se desatiende la calidad del producto periodístico en favor de una información más ligera y sometida a la dictadura de las audiencias. Las sospechas de que las formulaciones periodísticas protagonizadas por ciudadanos puedan esconder en muchas ocasiones "señuelos del peor populismo con el que los medios a la moderna laven su imagen ante unos ciudadanos acomodaticios" (Dader, 2010) han despertado mayoritariamente preocupación (Poell y van Dijk, 2014), por cuanto puede afectar a la independencia periodística, condición fundamental para el distanciamiento que confiere al periodista cierto nivel de autoridad y credibilidad.

\subsection{Las interrelaciones entre la conversación en redes sociales y la agenda-building}

Las exploraciones sobre el poder de los asuntos surgidos de espacios externos como las redes sociales ha propiciado un sugerente objeto de investigación, centrado en las interrelaciones que se establecen entre redes sociales y medios de comunicación. Tandoc Jr. y Vos (2015) sugieren que el creciente uso de medios sociales por parte de los periodistas les ha conducido a tener que equilibrar las normas y valores propios del periodismo con una creciente influencia de la audiencia, que es percibida como un elemento clave para la supervivencia de la actividad periodística.

En este sentido, Twitter se ha convertido en un nodo relevante de comunicación espontánea entre ciudadanos, en liza con la comunicación estratégica de actores sociales y políticos que ejercitan una amplia libertad para compartir contenidos alrededor de sus proyectos e intereses, como forma de poder (Castells, 2009). En ese ecosistema, la estructuración temática basada en términos y hashtags actúa como una 'folksonomía' que ayuda a organizar conversaciones entre usuarios y delinea una agenda de temas y protagonistas populares que son visibilizados con una fugacidad variable. En lo relativo al potencial de esta red social en la configuración de la agenda mediática, López Meri (2015, p. 37-38) sintetiza que la red social ha llegado a convertirse en un criterio de noticiabilidad más, cuyos debates, enfoques y fuentes afectan al proceso de newsmaking.

En el ámbito español, Rubio García (2014) constata la sintonía existente entre la agenda del público en Twitter y la agenda de los medios tradicionales debido al parejo acceso que profesionales y ciudadanos tienen a la información, mientras que Antón Crespo y Alonso del Barrio (2015) señalan que política, cultura y deporte suelen ser los temas que más coincidencias generan entre la agenda de portada de varios cibermedios españoles y la de los usuarios de esta red social. Otro estudio circunscrito al ámbito nacional revela que los cibermedios españoles mantienen una influencia moderada, pues la correspondencia entre las breaking news ofertadas por los diarios digitales y los asuntos que fueron trending topics de Twitter nunca superó el 25\% (Tous Rovirosa, Rivero Santamarina, Meso Ayerdi y Larrondo Ureta, 2015).

A la vista de estos antecedentes teóricos, partimos del objetivo de analizar las interrelaciones entre la actividad participativa de la audiencia y la agenda periodística de cuatro cibermedios generalistas líderes en el ámbito español (El País, El Mundo, El Confidencial y El Diario). Las tres hipótesis de esta investigación quedan enunciadas de la siguiente manera:

H1. Los principales temas presentes en las portadas web de los cibermedios líderes en España gozan de una atención profesional más duradera cuando coinciden con las agendas de temas modeladas por la actividad de la audiencia (clasificaciones de 'lo más leído' en sus sitios web y trending topics en Twitter). 
H2. Prevalece la brecha informativa que habitualmente separa los tratamientos informativos duros preferidos por los periodistas frente a los blandos predilectos para los lectores.

H3. En consecuencia, los asuntos más leídos, habitualmente blandos, logran unas métricas participativas más elevadas en forma de comentarios y tiempo como trending topics en Twitter que el resto de temas.

\section{Metodología}

Una vez enunciadas las hipótesis, la muestra estratégica de medios digitales estuvo formada por cuatro cibermedios líderes de audiencia en el ámbito español, que permitían aprovechar la participación y el feedback ciudadano, tanto desde el punto de vista de recursos disponibles como por la actividad potencial de un número crítico de usuarios y lectores. La selección de los casos se realizó a partir de los datos de audiencia ofrecidos por el proveedor de servicios de medición de audiencia, comScore en mayo de 2017, el mes previo a la recogida de datos. Para aportar diversidad y cierta dimensión comparativa, se seleccionaron los dos principales cibermedios de matriz impresa (ElPais.com y ElMundo.es) y los dos cibermedios nativos líderes (ElConfidencial.com y Eldiario.es).

\subsection{Población y muestra}

Para comprobar el grado de afinidad o interrelación existente entre los asuntos y tratamientos informativos presentes en la agenda de portada de los cibermedios líderes en España y las preferencias temáticas visibilizadas por parte de la audiencia mediante lecturas, comentarios y conversaciones sostenidas en Twitter se procedió a registrar diferentes datos de tipo cuantitativo. Por un lado, se realizó un seguimiento de las portadas de los cuatro cibermedios durante los 15 primeros días de junio de 2017, que fueron archivadas junto con las clasificaciones de 'lo más leído'. Por otro, se conservó el primer nivel de sus hipervínculos, lo que nos permitió contar con un universo de estudio formado por contenidos enriquecidos con enlaces completamente funcionales.

La unidad de análisis fue el tema informativo, que engloba uno o varios asuntos de interés noticioso vinculados entre sí y a menudo articulados en varias piezas (como noticias vinculadas, despieces o artículos de opinión) subsumidas en el tema principal. El análisis de este material se llevó a cabo seleccionando una muestra por cuotas (no probabilística) de los primeros 10 temas presentes en dos espacios del cibermedio: la portada y el ranking de 'lo más leído'. A partir de esta selección, se identificaron dos variables relevantes para el estudio: la importancia concedida por los propios profesionales de los cibermedios a los temas, así como el interés de la ciudadanía por ellos en forma de preferencia de lectura.

Así, en cada registro se seleccionaron los 10 temas cuyo posicionamiento denotaba mayor relevancia en portada (jerarquizados por sentido y gravedad de lectura, de izquierda a derecha y de arriba a abajo). De forma similar, del ranking de piezas más leídas también se tomaron las 10 primeras representadas en lista. Se registraron los temas de interés informativo con una frecuencia que reflejara los ritmos de actualización propios de la pauta informativa en España fijado en tres recogidas diarias $(9: 00$ h., 15:00 h. y 21:00 h. UTC+2). Por tanto, la muestra estratégica total $(n)$ ascendió a un total de 3.600 temas informativos (1.800 de portada y 1.800 de 'lo más leído').

\subsection{Instrumentos de recogida de información}

Con esta muestra se diseñó una plantilla de análisis que permitiese una comparación efectiva entre los contenidos de ambas agendas, revelando el grado de correspondencia de las propuestas 
informativas de cada uno de estos cibermedios con los intereses de sus audiencias y con el segmento de ciudadanía activa y conectada cuyas conversaciones se visibilizan en los comentarios de cada pieza y en la red social Twitter. Para la categorización de los temas partimos de las 16 categorías temáticas del estudio previo de agenda en cibermedios elaborado por Odriozola Chené (2012) y basado en los criterios del International Press and Telecommunications Council para la transferencia internacional de noticias, que adaptamos a nuestra investigación hasta alcanzar la veintena de temas:

Tabla 1. Hoja de codificación para analizar las interrelaciones entre la agenda profesional y las perfiladas por la actividad participativa de la ciudadanía en los sitios web de los cibermedios.

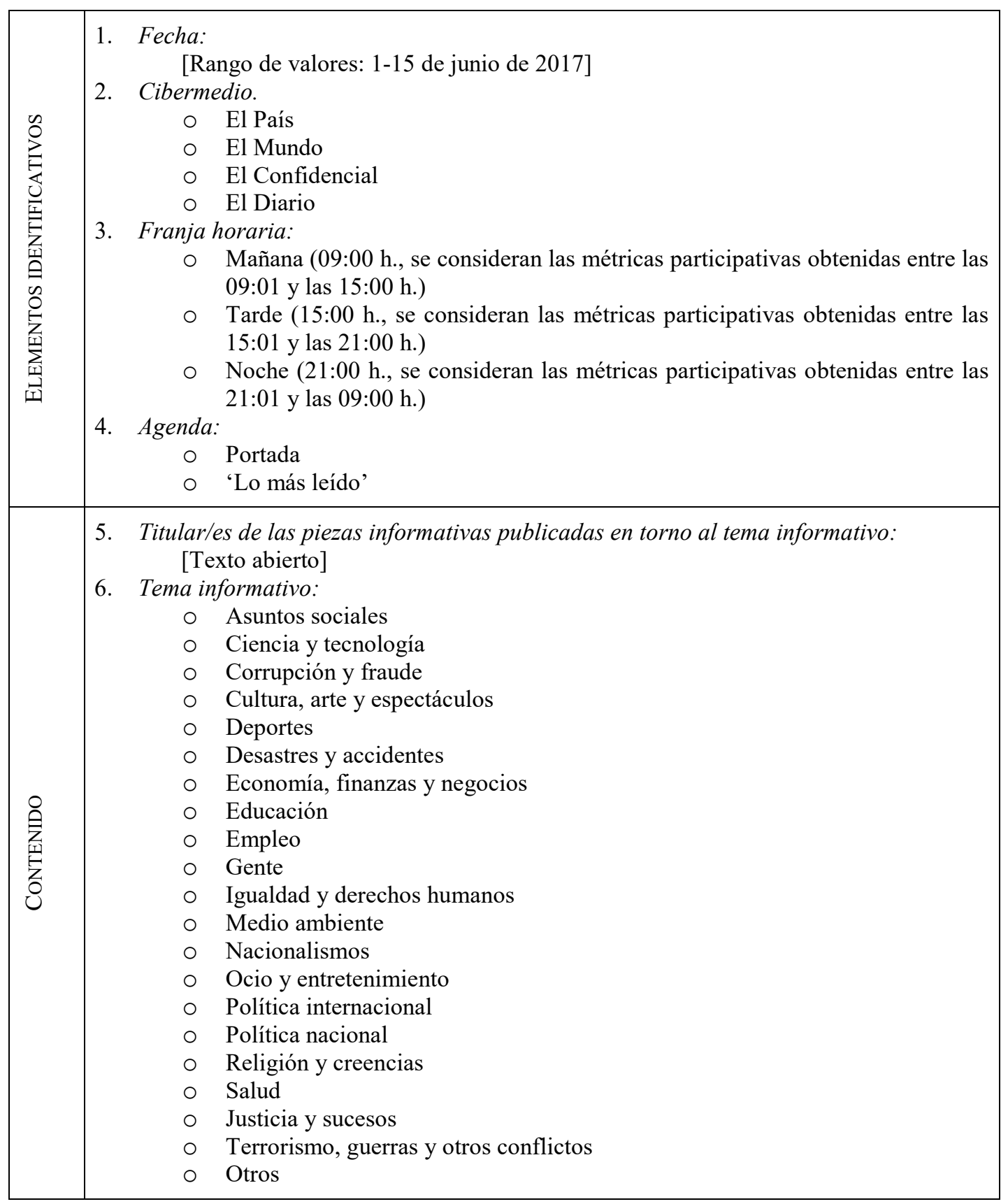


RLCS, Revista Latina de Comunicación Social, 75, 225-244

[Investigación] DOI: 10.4185/RLCS-2020-1424| ISSN 1138-5820 | Año 2020

\begin{tabular}{|c|c|}
\hline 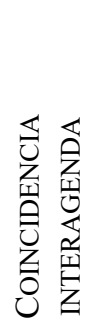 & $\begin{array}{l}\text { 7. Al menos una pieza informativa englobada en el tema está presente en la otra agenda } \\
\text { del sitio web del cibermedio (existe coincidencia entre portada y 'lo más leido'): } \\
\circ \text { Sí } \\
\circ \text { No } \\
\text { 8. Al menos un término englobado en el tema está presente en la agenda de 'temas del } \\
\text { momento' que conforma la conversación social de Twitter en España: } \\
\circ \text { Sí } \\
\circ \text { No }\end{array}$ \\
\hline Z๐ & $\begin{array}{l}\text { 9. Posición en la agenda: } \\
\text { [Rango de valores: 1-10] } \\
\text { 10. [Si } 4=\text { Portada] Permanencia del tema informativo en la siguiente franja horaria: } \\
\text { O Permanece } \\
\text { ○ Desaparece }\end{array}$ \\
\hline 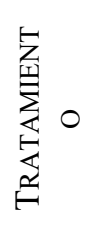 & $\begin{array}{l}\text { 11. Tratamiento informativo del tema: } \\
\begin{aligned} & \text { Duro } \\
& \text { Blando }\end{aligned}\end{array}$ \\
\hline 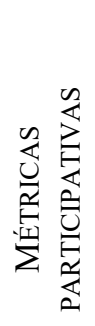 & $\begin{array}{l}\text { 12. [Si } 8 \text { = Sí] Minutos de conversación acumulados por los términos asociados al tema } \\
\text { que se alzaron como 'temas del momento' en Twitter (España): } \\
\text { [Rango de valores } \\
\text { 13. Comentarios generados por la pieza principal englobada en el tema: } \\
\text { [Rango de valores: de } 0 \text { a tantos como comentarios genere la pieza principal } \\
\text { englobada en el tema] }\end{array}$ \\
\hline
\end{tabular}

Fuente: elaboración propia.

La posición ocupada por cada tema en las dos agendas se empleó como variable crítica para comprobar la priorización. Por su parte, la atención sostenida a los temas (su permanencia) por parte de los periodistas ofrecía datos clave para abordar las interdependencias entre agendas. La coincidencia interagenda entre la agenda de 'lo más leído' y la agenda de portada se determinó comparando las piezas de la primera con las de la segunda en el momento de la recogida y en la franja horaria previa.

A la vista de los estudios precedentes, se optó por comprobar si el distanciamiento entre la predilección del juicio profesional por las noticias duras y el interés del público en las noticias blandas prevalecía. Siguiendo a Reinemann, Stanyer, Scherr y Legnante (2012), concluimos que la aproximación para determinar si un tema había sido tratado como hard o soft news debía ser multidimensional. Por tanto, determinamos la 'dureza' de cada tema informativo prestando atención a diversos factores en una tríada de dimensiones: el asunto (políticamente relevante/no relevante políticamente), el enfoque (su relevancia social/individual y su encuadre temático/episódico) y el estilo (la cobertura empleada es impersonal/personal y presenta una orientación no emocional/emocional). De tal modo que la orientación que dominase en dos o más de estas dimensiones determinó esta 'dureza', para que las categorías duro/blando fuesen recíprocamente excluyentes. 
Para medir la cercanía de los temas que eran objeto de cobertura en los cibermedios con los asuntos que interesaban al público, se relacionaron las dos agendas estudiadas en cada cibermedio con dos métricas participativas: el número de comentarios que suscitaban y la cantidad de tiempo que sus términos asociados se mantenían como 'temas del momento' en Twitter. Los temas más populares de esta plataforma durante cada jornada fueron obtenidos del listado que ofrece la sección española de Trendinalia ${ }^{1}$, que registra todos los términos y hashtags que alcanzan la condición de tendencia o 'tema del momento' nacional junto con la cantidad de tiempo que han permanecido en esta red social. Esta labor requirió una minuciosa organización del corpus de trending topics nacionales para rescatar aquellos relacionados semánticamente con cada tema aparecido en la muestra de los cuatro cibermedios. Fue necesario realizar un seguimiento exhaustivo de la actualidad de aquellos días para evitar la pérdida de información. Tras esta labor, se cuantificó la intensidad del debate despertado por cada uno de los 3.600 temas de la muestra mediante el cómputo del número total de minutos de conversación diarios asociados.

Por último, se comprobó la fiabilidad intercodificadores mediante la recodificación de una submuestra aleatoria del $15 \%$ de las 3.600 unidades de análisis por parte de un codificador externo y entrenado (Neuendorf, 2002; Igartua Perosanz, 2006). Las coincidencias obtenidas otorgaron una fiabilidad adecuada a los resultados del estudio, tanto en las variables cualitativas $(\overline{\mathrm{x}}$ de $\mathrm{AO}=0,91 ; \overline{\mathrm{x}}$ de $p i$ de Scott $=0,7)$ como en las cuantitativas $(\overline{\mathrm{x}}$ de $r$ de Pearson $=0,84)$.

\section{Resultados}

Los resultados del análisis de contenido de las interrelaciones entre la propuesta de temas diseñada por los profesionales del periodismo y la descrita por los intereses ciudadanos se estructuran a partir de los cinco objetivos relevantes de nuestro estudio: la selección de los temas y la coincidencia interagenda, la evolución y permanencia de estos asuntos en la agenda, el tratamiento informativo que recibieron y las métricas participativas asociadas.

\subsection{Selección de temas}

En líneas generales, el estudio comparativo de las dos agendas -de portada y 'lo más leído'- presentes en los cuatro cibermedios revela plena afinidad en los siete primeros tópicos que encabezan los temas más frecuentes propuestos por los periodistas y aquellos más leídos por la audiencia. Estos temas supusieron el $72,2 \%$ de los temas de portada y el $65,3 \%$ del total de los asuntos más leídos. Los dos primeros temas (política nacional y economía) coincidieron incluso en posición. Sin embargo, mientras los profesionales priorizaron las informaciones sobre corrupción y política internacional, los ciudadanos prefirieron el terrorismo y otros conflictos, además de los sucesos y los asuntos judiciales.

Los deportes también fueron un tema destacado, aunque su proporción en la agenda de 'lo más leído' por parte de la audiencia fue mayor que su presencia en la agenda de portada. Otros temas que experimentaron mayor protagonismo en la agenda de 'lo más leído' que en portada fueron el terrorismo y otros conflictos, la justicia y los sucesos, mientras que la política internacional recibió prácticamente la mitad de atención ciudadana que periodística. El ocio y el entretenimiento, que ocupa la octava posición en la agenda de temas más leídos, se desploma hasta el puesto $18 .^{\circ}$ de los temas de portada. Lo contrario sucede en el caso de los nacionalismos, relevantes en portada ( $8 .^{\mathrm{a}}$ posición) pero menos en el ranking de 'lo más leído' (12. posición).

\footnotetext{
${ }^{1}$ Accesible desde: http://www.trendinalia.com/twitter-trending-topics/spain/spain-today.html
} 
Si observamos las agendas de cada cibermedio por separado (figura 1), los temas prioritarios para El País fueron la política internacional, el terrorismo y otros conflictos y los deportes, mientras que sus lectores mostraron mayor interés por el terrorismo y otros conflictos, los asuntos judiciales y los sucesos, y la política internacional. Por su parte, los temas más relevantes para ElMundo.es fueron la política nacional, el terrorismo e internacional, mientras que sus lectores prefirieron el terrorismo, asuntos judiciales y los sucesos, y los deportes.

Entre los temas más destacados en ElConfidencial.com se encontraban la economía, la corrupción y la política nacional, mientras que sus lectores mostraron semejante predilección por la economía, pero también por el terrorismo y otros conflictos, los deportes y el ocio. Los temas más relevantes para la redacción de Eldiario.es fueron la política nacional, la corrupción y los asuntos económicos, mientras que sus lectores coincidieron plenamente con esos mismos asuntos, junto con la igualdad y los derechos humanos. 


\section{EL PAIS}

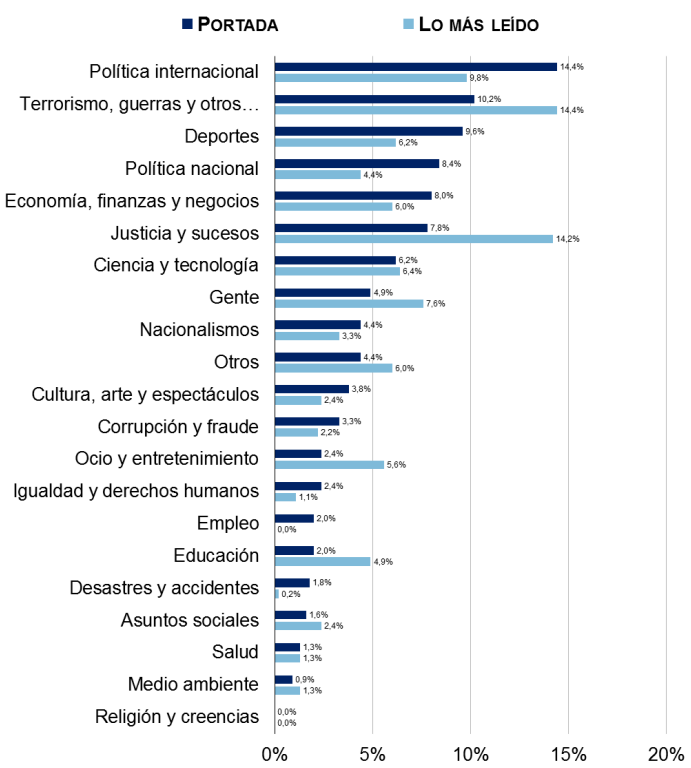

\section{El Confidencial}

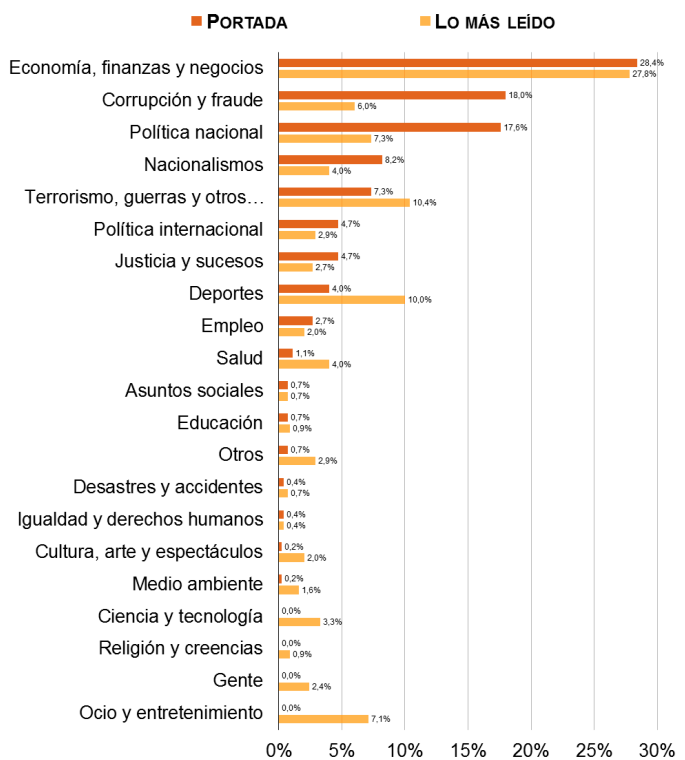

\section{EL年MUNDO}

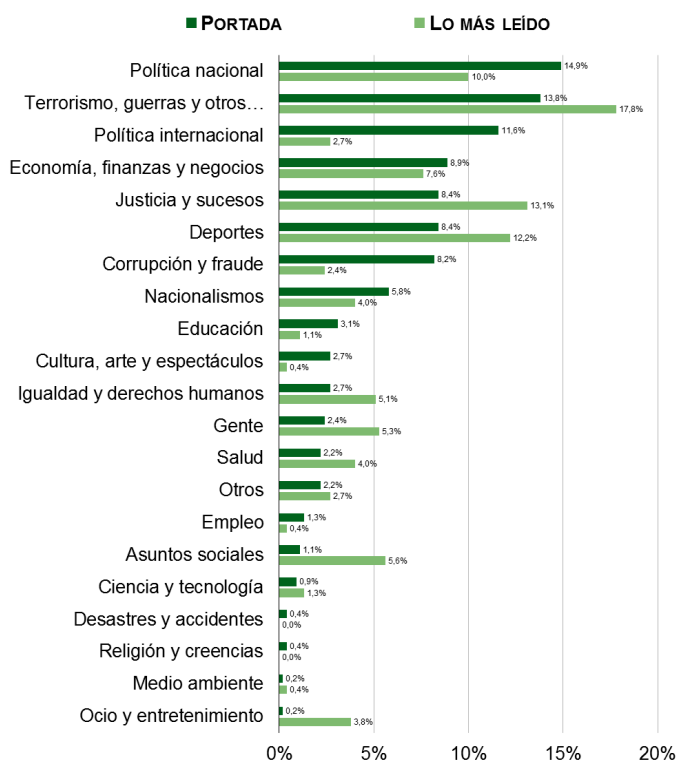

Oeldiario.es

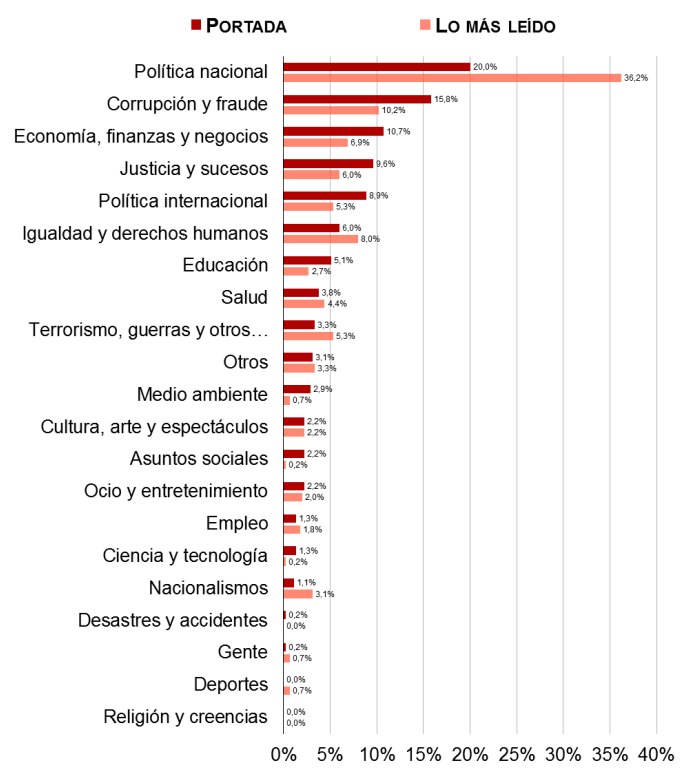

Figura 1: frecuencias de presencia de los temas en las agendas de portada y 'lo más leído' de cada cibermedio.

Fuente: elaboración propia.

\subsection{Coincidencia interagenda}

Los temas de las cuatro portadas estudiadas muestran un 42,1\% de coincidencia con la agenda de 'lo más leído' y un 44,8\% con los trending topics de Twitter. Comparativamente, la agenda de 'lo más leído' de los cuatro cibermedios presenta mayor coincidencia con los temas de sus respectivas portadas $(67,1 \%)$ y una similar con los 'temas del momento' de Twitter $(43,2 \%)$. Como se aprecia en 
la tabla 2, los cibermedios que más coincidencia interagenda logran entre los asuntos de sus agendas de portada y 'lo más leído' son El Diario y El País (47,8\% y 46,4\%, respectivamente). Por su parte, esos mismos contenidos de portada en El Mundo (37,3\%) y El Confidencial (36,9\%) suscitan un menor interés por parte de sus lectores.

Estos datos revelan que los lectores de El País son los que componen la agenda de temas más leídos que mejor encajan con los propuestos por los profesionales: aproximadamente tres de cada cuatro contenidos de 'lo más leído' han sido publicados entre los diez principales temas de portada. Por su parte, los lectores de El Confidencial coinciden menos con la oferta principal de portada del medio (solo un 56,9\% de las piezas más leídas pertenecen a temas presentes en los primeros puestos de portada).

Tabla 2. Proporción de temas que presentan coincidencia entre las agendas de portada y de 'lo más leido' de cada cibermedio.

\begin{tabular}{|c|c|c|c|c|c|c|c|c|c|}
\hline & & \multicolumn{2}{|c|}{ EL PAIS } & \multicolumn{2}{|c|}{ EL丰MUNDO } & \multicolumn{2}{|c|}{ El Confidencial } & \multicolumn{2}{|c|}{ O)eldiario.es } \\
\hline & & Portada & $\begin{array}{l}\text { 'Lo más } \\
\text { leído' }\end{array}$ & Portada & $\begin{array}{l}\text { 'Lo más } \\
\text { leído' }\end{array}$ & Portada & $\begin{array}{l}\text { 'Lo más } \\
\text { leído' }\end{array}$ & Portada & $\begin{array}{l}\text { 'Lo más } \\
\text { leído' }\end{array}$ \\
\hline \multirow{4}{*}{$\begin{array}{l}\text { COINCIDENCIA } \\
\text { PORTADA-'LO } \\
\text { MÁS LEÍDO' }\end{array}$} & Sí & $46,4 \%$ & $75,3 \%$ & $37,3 \%$ & $67,6 \%$ & $36,9 \%$ & $56,9 \%$ & $47,8 \%$ & $68,4 \%$ \\
\hline & No & $53,6 \%$ & $24,7 \%$ & $62,7 \%$ & $32,4 \%$ & $63,1 \%$ & $43,1 \%$ & $52,2 \%$ & $31,6 \%$ \\
\hline & Total & $100,0 \%$ & $100,0 \%$ & $100,0 \%$ & $100,0 \%$ & $100,0 \%$ & $100,0 \%$ & $100,0 \%$ & $100,0 \%$ \\
\hline & & \multicolumn{2}{|c|}{$\begin{array}{l}\text { Sig. } \chi^{2}: p_{v}=0,000 \\
<\alpha=0,05 \Rightarrow S e \\
\text { rechaza } H_{0}\end{array}$} & \multicolumn{2}{|c|}{$\begin{array}{l}\text { Sig. } \chi^{2}: p_{v}=0,000 \\
<\alpha=0,05 \Rightarrow S e \\
\text { rechaza } H_{0}\end{array}$} & \multicolumn{2}{|c|}{$\begin{array}{l}\text { Sig. } \chi^{2}: p_{v}=0,000 \\
<\alpha=0,05 \Rightarrow S e \\
\text { rechaza } H_{0}\end{array}$} & \multicolumn{2}{|c|}{$\begin{array}{l}\text { Sig. } \chi^{2}: p_{v}=0,000 \\
<\alpha=0,05 \Rightarrow S e \\
\text { rechaza } H_{0}\end{array}$} \\
\hline
\end{tabular}

Fuente: elaboración propia.

En lo referente a la presencia de términos asociados a los temas de portada en Twitter, El Mundo es el medio que más temas con trascendencia 'social' generó, dado que la práctica mitad de los asuntos propuestos en su portada $(49,3 \%)$ obtuvo algún tipo de repercusión social en forma de trending topics, algo que El País logró en términos similares (46,7\%). Por su parte, la agenda conformada por lo más leído convierte a los lectores de El Diario en la audiencia que mejor delinea qué temas producen conversación en redes sociales $(53,1 \%)$, a cierta distancia de las cifras que componen los temas más leídos en El Mundo (44,4\%), El País (39,8\%) y El Confidencial (35,3\%).

Tabla 3. Proporción de temas que presentan coincidencia con los 'temas del momento' en Twitter en las agendas de portada y de 'lo más leído' de cada cibermedio.

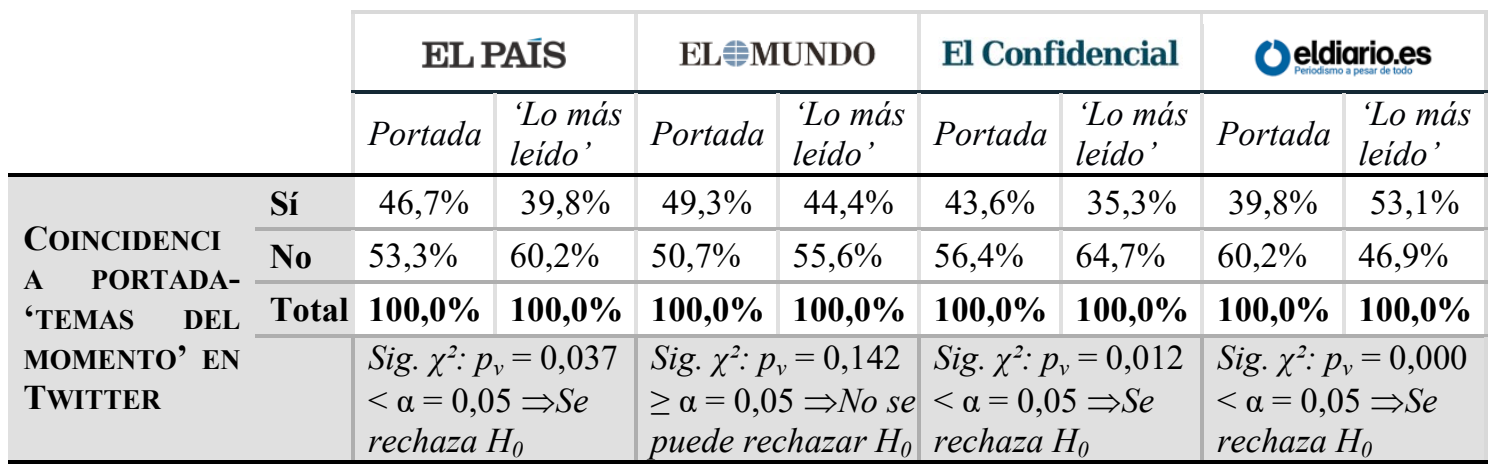

Fuente: elaboración propia. 


\subsection{Evolución de los temas}

Para medir la relación existente entre el juicio profesional (agenda de portada) y el feedback inmediato que representan los contenidos más visitados (agenda de 'lo más leído'), los comentarios generados y la conversación aparejada a ellos en redes sociales se determinó la evolución de los temas de portada al avanzar entre franjas horarias contiguas, para comprobar si el tema permanecía (subía, bajaba o mantenía su posición) o desaparecía de portada. Este dato arrojó luz sobre la atención sostenida y la continuidad en el foco que los profesionales mantienen sobre los temas de su agenda.

Los datos demuestran que, de los 1.800 temas recogidos en las portadas de los cibermedios, solo 683 (un 37,9\%) recibieron seguimiento en la siguiente franja horaria - no desaparecieron-. La atención profesional más prolongada la recibieron el terrorismo, la política nacional, la economía y los nacionalismos. Precisamente, estos tres primeros temas coincidieron con los tres primeros del ranking de 'lo más leído' como ya se apuntó. Asuntos como la religión, la igualdad, los desastres y accidentes, el medio ambiente, los asuntos sociales y la crónica social apenas consiguieron fijarse en la agenda de portada.

De los 683 temas que permanecieron en portada de una franja horaria a la siguiente, un $60,3 \%$ además se asociaba a términos que figuraron entre los trending topics de Twitter y un 62,1\% contenía piezas que formaron parte de lo más leído, lo que muestra la notable relación entre el feedback ciudadano sobre determinados asuntos y una correspondiente atención periodística hacia los mismos (y a la inversa). Entre esos temas con una atención profesional más permanente destacan cinco asuntos: terrorismo y otros conflictos, economía, política nacional, política internacional y nacionalismos. Los cuatro primeros coinciden con los temas que los cibermedios abordaban de forma más frecuente.

De los temas ofrecidos en portada cuyas piezas se auparon entre lo más leído de los cuatro cibermedios, obtuvieron una atención profesional más permanente la economía, la política nacional, el terrorismo y otros conflictos, la cultura, los nacionalismos, la corrupción e internacional. De ellos, cultura y nacionalismos fueron temas tratados con menor frecuencia en la agenda de portada y, sin embargo, lograron mayor permanencia en la oferta profesional de las cabeceras. Por último, los datos revelaron que la ubicación de los temas en portada también mantenía correspondencia con la permanencia de estos, de tal modo que, cuanto más alta resultaba la posición de un tema, más frecuentemente permanecía este tema en el tránsito de una franja horaria a la siguiente.

\subsection{Tratamiento informativo de los temas}

Al observar cómo se distribuyen las frecuencias de tratamientos empleados en los temas de los cuatro cibermedios, se aprecia que, mientras el conjunto de portadas acogió una representación equilibrada de temas duros $(50,7 \%)$ y blandos $(49,3 \%)$, las agendas de 'lo más leído' por la audiencia mostraron cierta propensión a los tratamientos blandos (67,3\%). Mientras que El Mundo y El Diario se ajustaron al mencionado equilibrio de tratamientos, El País mostró propensión a los temas blandos $(60,44 \%)$ y El Confidencial, a los duros $(60,89 \%)$.

Entre los temas que más habitualmente entraron en la categoría de hard news se encontraban la política internacional y nacional, la corrupción y los nacionalismos. Los asuntos económicos alternaban las presentaciones hard y soft, algo que también sucedió con el empleo y los desastres naturales. 
Además, los resultados demostraron que los contenidos duros superaron a los blandos en cuanto a su evolución y permanencia en portada (62,2\% frente a 37,8\%), lo que confirma la mayor adherencia de estos tratamientos a la agenda propuesta por el medio. La posición del contenido en la portada del cibermedio también ofreció pistas inequívocas sobre la propensión de los profesionales a priorizar tratamientos duros en esa agenda. Así, cuanto más elevada es la posición que ocupa el tema en la agenda de portada, más probabilidades existen de que este reciba un tratamiento duro, y a la inversa.

En todas las posiciones del ranking de temas más leídos, los contenidos blandos siempre se impusieron en proporción a los duros, lo que consecuentemente confirma la disonancia entre el tratamiento de los temas que los profesionales seleccionan y el de los asuntos que sus audiencias prefieren leer, ver o escuchar.

\subsection{Métricas participativas asociadas a los temas}

El Mundo y El País fueron los cibermedios cuyos temas de portada conectaron de forma más prolongada con los términos y hashtags que conformaban los 'temas del momento' en Twitter. El Confidencial y, especialmente, El Diario obtuvieron cifras más discretas. El País destaca con el mayor número de comentarios generados, seguido de El Mundo y El Diario (relativamente cercanos entre sí), y El Confidencial.

Tabla 4. Valores agregados y medianos de los minutos como trending topics en Twitter asociados y los comentarios generados por los temas presentes en la agenda de portada de cada cibermedio.

\begin{tabular}{|c|c|c|c|c|c|c|c|c|}
\hline & \multicolumn{2}{|c|}{ EL PAİS } & \multicolumn{2}{|c|}{ EL事MUNDO } & \multicolumn{2}{|c|}{ El Confidencial } & \multicolumn{2}{|c|}{ Oeldiario.es } \\
\hline & Portada & & Portada & & Portada & & Portada & \\
\hline & $\begin{array}{l}\text { Suma } \\
(\Sigma)\end{array}$ & $\begin{array}{l}\text { Mediana } \\
(M d n)\end{array}$ & $\begin{array}{l}\text { Suma } \\
(\Sigma)\end{array}$ & $\begin{array}{l}\text { Mediana } \\
(M d n)\end{array}$ & $\begin{array}{l}\text { Suma } \\
(\Sigma)\end{array}$ & $\begin{array}{l}\text { Mediana } \\
(M d n)\end{array}$ & $\begin{array}{l}\text { Suma } \\
(\Sigma)\end{array}$ & $\begin{array}{l}\text { Mediana } \\
(M d n)\end{array}$ \\
\hline $\begin{array}{l}\text { MINUTOS COMO } \\
\text { TRENDING } \\
\text { TOPICS EN } \\
\text { TWITTER } \\
\end{array}$ & 206.300 & 505 & 207.999 & 490 & 191.530 & 463 & 155.695 & 470 \\
\hline COMENTARIOS & 351.334 & 124 & 115.018 & 63 & 18.824 & 13 & 59.464 & 46 \\
\hline
\end{tabular}

Fuente: elaboración propia.

Los temas duros lograron, en todos los diarios, más minutos como trending topics en Twitter que los temas blandos, un dato que se aprecia tanto en la agenda de portada (530 frente a 440 minutos) como en la agenda de 'lo más leído' (695 frente a 520 minutos). Por su parte, los comentarios muestran un comportamiento similar: en ambas agendas se realizaron más comentarios cuando el tratamiento era duro que cuando este era blando. La prueba $U$ de Mann-Whitney confirma que existen diferencias estadísticamente significativas entre los dos tratamientos y los minutos de conversación (Sig. $=0,003$ ) así como entre los dos tratamientos y los comentarios que se les asocian $(\mathrm{Sig} .=0,000)$, siempre más altos para los contenidos duros.

Atendiendo a la temática y tratamiento de los temas analizados, existe cierto orden en los temas de portada cuyos términos asociados lograron más minutos de conversación en Twitter. Así, los temas habitualmente abordados como contenidos blandos, fueron los que lograron más minutos. Las únicas excepciones quedan representadas por temas duros como la política internacional, los nacionalismos 
y la política nacional que, aunque también obtuvieron valores medianos notables como trending topics, lo hicieron en menor medida que los anteriores. Sin embargo, al tener en cuenta los temas presentes en la agenda de 'lo más leído', observamos cómo, los temas que consiguieron más minutos en Twitter fueron aquellos repetidamente tratados como duros.

En lo relativo a los comentarios generados en función de cada temática recogida en las portadas, los asuntos políticos duros (nacionalismos y política nacional) fueron los que más comentarios fomentaron, si bien algunos contenidos blandos destacaron en cuanto a valores medianos (salud, igualdad, ocio, ciencia y tecnología y crónica social). Por su parte, los comentarios recogidos en la agenda de 'lo más leído' volvieron a subrayar la relevancia de los temas duros. Alrededor de tres de cada cuatro temas vieron incrementar sus valores medianos de comentarios respecto a los obtenidos por esos mismos asuntos en la agenda de portada.

Por su parte, los asuntos que permanecen en portada obtienen más minutos agregados y de mediana como 'temas del momento' en Twitter que aquellos que reciben una atención menos sostenida y desaparecen. Asimismo, los valores medianos de minutos como trending topics de los asuntos que permanecen en portada llegan a aumentar un $22,1 \%$ si estos temas acceden a la agenda de lo más leído. La prueba $U$ de Mann-Whitney (Sig.=0,000) confirma que existen diferencias estadísticamente significativas entre la permanencia o evanescencia en portada y los minutos de conversación que se les asocian, siempre más altos para los contenidos que permanecen.

Tabla 5. Valores agregados y medianos de los minutos de conversación como trending topics en Twitter de los términos asociados a los temas presentes en la agenda de portada y aquellos temas de portada cuyas piezas coinciden con las de la agenda de 'lo más leído', según su permanencia en los cuatro cibermedios.

\begin{tabular}{|c|c|c|c|c|c|}
\hline & & \multicolumn{4}{|c|}{ MINUTOS COMO TRENDING TOPICS EN TWITTER } \\
\hline & & \multicolumn{2}{|l|}{ Portada } & \multicolumn{2}{|c|}{$\begin{array}{l}\text { Temas de portada cuyas piezas } \\
\text { coinciden con las de la agenda } \\
\text { de 'lo más leído' }\end{array}$} \\
\hline & & Suma $(\Sigma)$ & $\begin{array}{l}\text { Mediana } \\
(\text { Mdn) }\end{array}$ & Suma $(\Sigma)$ & $\begin{array}{l}\text { Mediana } \\
(\text { Mdn })\end{array}$ \\
\hline \multirow[b]{2}{*}{ Permanencia } & Permanece & 538.974 & 688 & 445.219 & 840 \\
\hline & Desaparece & 222.550 & 385 & 115.975 & 495 \\
\hline
\end{tabular}

Fuente: elaboración propia.

Del mismo modo, los asuntos que permanecen en portada logran mayor número de comentarios en términos medianos que aquellos que desaparecen. Así, las cifras medianas de comentarios en portada se incrementan un tercio $(33,3 \%)$ si los términos asociados al tema coinciden con trending topics de Twitter y hasta casi el doble $(82,2 \%)$ si alguna de sus piezas coincide con las presentes en la agenda de 'lo más leído'. 
RLCS, Revista Latina de Comunicación Social, 75, 225-244

[Investigación] DOI: 10.4185/RLCS-2020-1424| ISSN 1138-5820 | Año 2020

Tabla 6. Valores agregados y medianos de los comentarios generados por los temas presentes en la agenda de portada y por aquellos temas de portada coincidentes con los trending topics en Twitter y con 'lo más leído', según su permanencia en los cuatro cibermedios.

\begin{tabular}{|c|c|c|c|c|c|c|c|}
\hline & & \multicolumn{6}{|c|}{ COMENTARIOS } \\
\hline & & \multicolumn{2}{|l|}{ Portada } & \multicolumn{2}{|c|}{$\begin{array}{l}\text { Temas de portada } \\
\text { cuyos términos } \\
\text { asociados coinciden } \\
\text { con los trending } \\
\text { topics de Twitter }\end{array}$} & \multicolumn{2}{|c|}{$\begin{array}{l}\text { Temas de portada } \\
\text { cuyas piezas } \\
\text { coinciden con las de } \\
\text { la agenda de 'lo más } \\
\text { leído' }\end{array}$} \\
\hline & & Suma $(\Sigma)$ & $\begin{array}{l}\text { Mediana } \\
\text { (Mdn) }\end{array}$ & Suma $(\Sigma)$ & $\begin{array}{l}\text { Mediana } \\
\text { (Mdn) }\end{array}$ & $\operatorname{Suma}(\Sigma)$ & $\begin{array}{l}\text { Mediana } \\
\text { (Mdn) }\end{array}$ \\
\hline \multirow[b]{2}{*}{ Permanencia } & Permanece & 141.504 & 45 & 117.601 & 60 & 121.970 & 82 \\
\hline & Desaparece & 111.036 & 30 & 59.128 & 40 & 51.678 & 69 \\
\hline
\end{tabular}

Fuente: elaboración propia.

Finalmente, en cuanto a la franja horaria, los asuntos surgidos entre las 9:01 h. y las 15:00 h. lograron cifras sutilmente más elevadas que el resto de franjas del día.

\section{Discusión y conclusiones}

Los cibermedios estudiados presentan una agenda de portada sensible a la participación ciudadana que se traduce en un protagonismo más duradero para aquellos asuntos periodísticos que coinciden con los más leídos en el sitio web o los 'temas del momento' en Twitter. Los temas que los profesionales seleccionan reciben una atención periodística y un protagonismo más permanente cuando entran en sintonía con los asuntos que la participación ciudadana visibiliza mediante formas participativas ligeras -la agregación de las clasificaciones de 'lo más leído'- e incluso con los temas perfilados en espacios que propician una participación más densa -a través de métricas relacionadas con las conversaciones surgidas en los comentarios del cibermedio o las que cristalizan como trending topics en Twitter-.

Se puede considerar, por lo tanto, confirmada la primera hipótesis de esta investigación. Los temas presentes en las agendas de portada que permanecen hasta la siguiente franja horaria estudiada son más abundantes cuando sus términos asociados figuran entre los trending topics de Twitter, y la proporción se eleva aún más cuando sus piezas coinciden con 'lo más leído'. De los asuntos que permanecen en portada, la mayoría acaba fraguando como 'temas del momento' de Twitter o como asuntos más leídos. Expresado de modo inverso, los trending topics y los asuntos más leídos instigan temas de portada más perdurables que los asuntos que no alcanzan cualquiera de estas dos condiciones. Así, la mayoría de temas efímeros no logran ser 'temas del momento' ni ubicarse entre lo más leído.

Nuestro análisis revela que el conjunto de los cibermedios estudiados plantea una oferta temática de portada que encaja mayoritariamente con los temas más leídos por el cúmulo de sus audiencias. No obstante, a pesar de esta aparente correspondencia y homogeneización interagenda, cada uno de los cuatro diarios presenta ofertas informativas distintivas y acordes a sus señas identitarias y editoriales.

En lo relativo a la presencia de los temas en Twitter, los cibermedios de matriz impresa parecen influir más o afinar mejor su criterio de noticiabilidad de acuerdo a los intereses de los usuarios de esta red social, dado que la práctica mitad de los asuntos propuestos en sus portadas obtuvieron con 
algún tipo de repercusión social en forma de trending topics. La agenda conformada por lo más leído convierte a los lectores de El Diario en la audiencia que mejor delinea qué temas producen conversación en redes sociales, a cierta distancia de las cifras que componen los temas más leídos en El Mundo, El País y El Confidencial.

El Diario y El País son los cibermedios más efectivos al fijar la agenda de sus lectores, pues sus temas de portada son los que más se filtran a sus respectivas agendas de 'lo más leído'. Los lectores de El País delinean con sus lecturas la agenda que más coincidencias registra respecto a la de portada. Por su parte, la audiencia que define la agenda de 'lo más leído' en Eldiario.es se distingue por ser la que señala con mayor acierto qué asuntos se alzan como trending topics en Twitter.

En lo que se refiere a la segunda hipótesis, prevalece la brecha informativa que distancia los tratamientos informativos ofertados por los profesionales de los demandados por los ciudadanos. Los tratamientos del conjunto de agendas de portada perpetúan una nítida brecha informativa: el criterio profesional privilegia los contenidos duros mientras que los contenidos blandos se imponen en la agenda de 'lo más leído'.

Mientras que las portadas compensan equitativamente tratamientos duros y blandos, dos de cada tres asuntos de la agenda de 'lo más leído' son blandos. Nuestro estudio revela que la jerarquización o posición de cada tema de portada ofrece indicios sobre este tratamiento informativo: en las primeras posiciones de estas agendas los profesionales ubican temas más duros y, conforme se descienden puestos, los contenidos blandos ganan protagonismo. En contraste, los contenidos blandos son siempre más abundantes en todas las posiciones de las agendas de 'lo más leído'. De acuerdo con nuestros resultados, los asuntos que reciben tratamientos informativos duros permanecen y se afianzan mejor en la agenda de portada que los temas blandos.

Los temas duros logran métricas participativas más elevadas que los blandos. El tratamiento informativo se convierte así en un factor catalizador de la participación, en concomitancia con otros como la permanencia y posición en portada, la coincidencia con las agendas ciudadanas estudiadas y el horario de publicación. Son los temas duros los que en mayor medida capitalizan los comentarios y la conversación sobre el conjunto de asuntos de portada que se alzan como 'temas del momento' en Twitter. Por lo tanto, la tercera hipótesis propuesta queda refutada. Nuestros datos sostienen que un tema obtiene mejores métricas participativas cuando reúne una o varias de las siguientes condiciones: (1) recibe un tratamiento duro, (2) permanece en portada, (3) se sitúa en una posición elevada de la misma, (4) accede a la agenda de 'lo más leído', (5) coincide con los 'temas del momento' en Twitter o (6) se publica en la franja horaria de mañana. La combinación de varias de estas circunstancias contribuye acumulativamente a multiplicar estas cifras participativas.

Tanto en la agenda de portada como en la de 'lo más leído' los temas que reciben un tratamiento informativo duro suman más comentarios y minutos de conversación en Twitter agregados y medianos que los temas blandos, especialmente en la agenda de 'lo más leído' propensa a los tratamientos blandos. Los temas de portada que consiguen mejores medidas como trending topics son asuntos sociales, deportes y gente (temas que reciben tratamientos blandos), lo que resulta paradójico en una agenda controlada por profesionales que equilibran tratamientos duros y blandos. No resulta menos sorprendente que en la agenda de 'lo más leído' los temas que logran las mayores cifras medianas de minutos como 'temas del momento' en Twitter sean típicamente duros, como la economía o la política internacional.

En cuanto a los comentarios recogidos en portada, temas duros como los nacionalismos y la política son los que suscitan mayor número de comentarios, por lo que pueden ser considerados los 
principales impulsores del debate en los cibermedios estudiados. Concretamente, las agendas de portada propuestas por los dos cibermedios de matriz impresa analizados, El País y El Mundo, son las que conectan durante más minutos con los términos y hashtags de los 'temas del momento' en Twitter. Igualmente, ambos diarios presentan las cifras medianas más elevadas de comentarios en sus temas de portada, lo que da muestra de sus amplias comunidades de comentaristas.

Los profesionales deberían evaluar con interés los factores de catalización participativa en la construcción de su agenda informativa, dado que, a la vista de los datos obtenidos sobre métricas participativas, los tratamientos informativos duros que más se acomodan a los criterios profesionales sí que honran los intereses de la audiencia que conversa a través de comentarios en el sitio web del cibermedio o en Twitter. Por tanto, aunque la brecha informativa sigue presente con la generalidad de los lectores, nuestros resultados respaldan la idea de que el criterio profesional tiende puentes que salvan parcialmente este gap, al menos con la fracción más activa de la audiencia.

\section{Referencias}

Abbott, J. Y. (2017). Tensions in the scholarship on participatory journalism and citizen journalism, Annals of the International Communication Association, 41(3-4). https://doi.org/10.1080/23808985.2017.1350927

Antón Crespo, M. y Alonso del Barrio, E. (2015). El 'trending topic' frente a la 'agenda setting'. Estudios sobre el Mensaje Periodístico, 21 (núm. esp.), 23-34. https://doi.org/10.5209/rev_ESMP.2015.v21.51125

Boczkowski, P. J. y Mitchelstein, E. (2015). La brecha de las noticias: La divergencia entre las preferencias informativas de los medios y el público. Manantial.

Castells, M. (2009). Comunicación y poder. Alianza.

Dader, J. L. (2010, 30 de junio). La débil identidad del periodismo en la hipermodernidad. Nueva Revista. https://www.nuevarevista.net/revista-sociedad/la-debil-identidad-del-periodismo-en-lahipermodernidad/

Lawrence, R. G., Radcliffe, D. \& Schmidt, T. R. (2017). Practicing Engagement: Participatory journalism in the Web 2.0 era. Journalism Practice.

https://doi.org/10.1080/17512786.2017.1391712

Lee, A. M., Lewis, S. C. \& Powers, M. (2012). Audience Clicks and News Placement: A Study of Time-Lagged Influence in Online Journalism. Communication Research, 41(4), 505-530. https://doi.org/10.1177/0093650212467031

López Meri, A. (2015). Twitter como fuente informativa de sucesos imprevistos: el seguimiento de hashtags en el caso \#ArdeValencia. Anuario electrónico de estudios en Comunicación Social "Disertaciones", 8(1), 27-51. https://doi.org/10.12804/disertaciones.01.2015.02

Manosevitch, I. \& Tenenboim, O. (2017). The Multifaceted Role of User-Generated Content in News Websites: An analytical framework. Digital Journalism, 5(6), 731-752. https://doi.org/10.1080/21670811.2016.1189840 
Masip, P., Guallar, J., Suau, J. y Ruiz-Caballero, C. (2015). Información de actualidad y redes sociales: comportamiento de las audiencias. El profesional de la información, 24(4), 363-370. https://doi.org/10.3145/epi.2015.jul.02

Meraz, S. \& Papacharissi, Z. (2016). Networked Framing and Gatekeeping. En T. Witschge, C.W. Anderson, D. Domingo y A. Hermida (Eds.), The SAGE Handbook of Digital Journalism (pp. 95112). Sage.

Mitchelstein, E., Boczkowski, P. J., Wagner, C. y Leiva, S. (2016). La brecha de las noticias en Argentina: factores contextuales y preferencias de periodistas y público. Palabra Clave, 19(4), 1027-1047. https://doi.org/10.5294/pacla.2016.19.4.4

Morera Hernández, C. (2016). Lo más leído: infoentretenimiento, propaganda y anécdotas en la versión digital de los diarios españoles. Estudios sobre el Mensaje Periodístico, 23(1), 117-133. https://doi.org/10.5209/ESMP.55586

Newhagen, J. E. \& Levy, M. R. (1996). Distributed communication architectures and news. College of Journalism, University of Maryland.

Nguyen, A. (2013). Online news audiences: the challenges of web metrics. En S. Allan y K. FowlerWatt (eds.), Journalism: New Challenges (pp. 146-161). Poole: CJCR-Centre for Journalism \& Communication Research, Bournemouth University. http://eprints.bournemouth.ac.uk/20929/1/The\%20challenges\%20of\%20web\%20metrics.pdf

Nielsen, R. K. (2017). News media, search engines and social networking sites as varieties of online gatekeepers. En C. Peters y M. Broersma (Eds.), Rethinking Journalism Again: Societal role and public relevance in a digital age (pp. 81-96). Routledge.

Odriozola Chené, J. (2012). Cibermedios y "agenda-setting": la configuración de la agenda mediática internacional. Estudios sobre el Mensaje Periodístico, 18(1), 157-171. https://doi.org/10.5209/rev_ESMP.2012.v18.n1.39363

Pérez-Díaz, P. L., Berná Sicilia, C. \& Arroyas Langa, E. (2016). The conversation on political issues on Twitter: An analysis of the participation and frames in the debate on the 'Wert Law' and evictions in Spain. Obets, 11(1), 311-330. https://doi.org/10.14198/OBETS2016.11.1.12

Poell, T. \& Van Dijk, J. (2014). Social Media and Journalistic Independence. En J. Bennett y N. Strange (eds.), Media Independence: Working with Freedom or Working for Free? (pp. 182-201). Routledge.

Reinemann, C., Stanyer, J., Scherr, S. \& Legnante, G. (2012). Hard and Soft News: A Review of Concepts, Operationalizations and Key Findings. Journalism, 13(2), 221-239. https://doi.org/10.1177/1464884911427803

Rosen, J. (1997). Introduction: 'We will have that conversation': Journalism and democracy in the thought of James W. Carey. En E.S. Munson y C.A. Warren (Eds.), James Carey: A Critical Reader (pp. 191-206). The University of Minnesota Press. 
Rubio García, R. (2014). Twitter y la teoría de la Agenda-Setting: mensajes de la opinión pública digital. Estudios sobre el Mensaje Periodístico, 20(1), 249-264.

https://doi.org/10.5209/rev_ESMP.2014.v20.n1.45230

Shoemaker, P. J., Johnson, P. R., Seo, H. \& Wang, X. (2010). Readers as gatekeepers of online news: Brazil, China, and the United States. Brazilian Journalism Research, 6(1), 55-77. https://doi.org/10.25200/BJR.v6n1.2010.226

Singer, J. B., Domingo, D., Heinonen, A., Hermida, A., Paulussen, S., Quandt, T., et al. (2011). Participatory Journalism: Guarding Open Gates at Online Newspapers. Wiley-Blackwell.

Singer, J. B. (2014). User-generated visibility: Secondary gatekeeping in a shared media space. New Media \& Society, 16(1), 55-73. https://doi.org/10.1177/1461444813477833

Tandoc Jr., E. C. (2014). Journalism is twerking? How web analytics is changing the process of gatekeeping. New Media \& Society, 16(4), 559-575. https://doi.org/10.1177/1461444814530541

Tandoc Jr., E. C. \& Thomas, R. J. (2015). The Ethics of Web Analytics. Implications of using audience metrics in news construction. Digital Journalism, 3(2), 243-258.

https://doi.org/10.1080/21670811.2014.909122

Tandoc Jr., E. C. \& Vos, T. P. (2015). The journalist is marketing the news: Social media in the gatekeeping process. Journalism Practice, 10(8), 950-966.

https://doi.org/10.1080/17512786.2015.1087811

Tous Rovirosa, A., Rivero Santamarina, D., Meso Ayerdi, K. \& Larrondo Ureta, A. (2015). Ambient Journalism in Spain. How Twitter and NREs are redefining agenda setting in El País, El Mundo, La Razón, ABC and La Vanguardia. Trípodos, 36, 35-54. http://www.tripodos.com/index.php/Facultat_Comunicacio_Blanquerna/article/download/241/197

Welbers, K., van Atteveldt, W., Kleinnijenhuis, J., Ruigrok, N. \& Schaper, J. (2016). News selection criteria in the digital age: Professional norms versus online audience metrics. Journalism, 17(8), 1037-1053. https://doi.org/10.1177/1464884915595474

\section{AUTORES:}

\section{Pedro Luis Pérez Díaz}

Universidad Católica San Antonio de Murcia. España.

(Murcia, 1986) es Licenciado en Periodismo y Doctor en Comunicación por la Universidad Católica San Antonio de Murcia (UCAM), donde forma parte del Grupo de Investigación en Comunicación, Política e Imagen. Entre sus líneas de investigación ha privilegiado la mediación periodística en redes sociales y la participación en el periodismo digital, objeto de estudio de su tesis doctoral. Tras la obtención de una beca de formación del personal investigador de la Fundación Séneca, Agencia de Ciencia y Tecnología de la Región de Murcia, ha desarrollado su actividad docente e investigadora en distintos centros universitarios nacionales e internacionales de prestigio como la Universidad de Stanford (EE.UU.) y la Universidad de Florida (EE.UU.). Asimismo, ha participado en diversos proyectos I+D: uno regional competitivo financiado por la Fundación Séneca, vinculado con la comunicación política, las redes sociales y los jóvenes y otro nacional, relacionado con la rendición de cuentas y la transparencia en el ámbito de la administración local digital. Actualmente, es profesor 
en los grados en Comunicación y Turismo de la UCAM, además de ser el coordinador del máster en periodismo deportivo impartido en esta universidad.

plperez@ucam.edu

Índice $\mathrm{H}: 4$

Orcid ID: http://orcid.org/0000-0003-1104-0262

Google Scholar: http://scholar.google.es/citations?hl=es\&user=JkohSXAAAAAJ

\section{Enrique Arroyas Langa}

Universidad Católica San Antonio de Murcia. España.

(Valencia, 1966) es Licenciado en Ciencias de la Información por la Universidad de Navarra y Doctor en Comunicación por la Universidad Católica San Antonio de Murcia, con un sexenio de investigación activo reconocido por la CNEAI (2006-2014). Desde el año 2003 es profesor de la Facultad de Comunicación de la UCAM, donde además de impartir las asignaturas de Redacción Periodística y Periodismo Especializado, es el director del Título de Experto en Comunicación Política e Institucional. Cuenta con una trayectoria consolidada en el estudio de la mediación periodística en las redes sociales, habitualmente desde el prisma de la teoría del periodismo en su faceta de configurador de la opinión pública. Como miembro del Grupo de Investigación en Comunicación Política e Imagen, su línea de estudio se ha centrado en el papel del periodista como líder de opinión y la calidad del debate abierto a la participación del público (con artículos como 'The conversation on political issues on Twitter', 'Twitter y la prensa: dos relatos sobre la 'ley Wert' y 'Del des-contexto al descontento: agenda y discurso de la prensa durante las elecciones autonómicas en la Región de Murcia').

earroyas@ucam.edu

\section{Índice H: 4}

Orcid ID: http://orcid.org/0000-0001-6578-1571

Google Scholar: http://scholar.google.es/citations?user=9cZPGhAAAAAJ

\section{Rocío Zamora Medina}

Universidad de Murcia. España.

(Murcia, 1972), es profesora Titular de Universidad en el área de Periodismo, es Doctora en Comunicación Pública por la Universidad de Navarra y desde 2012 forma parte de la Facultad de Comunicación de la Universidad de Murcia, donde imparte docencia en Fundamentos de la Comunicación y de la Información. Fue profesora de Periodismo en la UCAM durante más de 15 años, donde ejerció el cargo de Vicedecana de Periodismo. Destaca por su experiencia docente internacional de grado y posgrado en más de 15 universidades europeas y americanas. Como investigadora, ha centra su investigación mayoritariamente en el análisis estratégico de campañas electorales en contextos online y sus efectos en la formación de opinión pública. Buena parte de sus investigaciones se recogen en más de una veintena de artículos publicados en revistas de impacto nacional e internacional, así como en medio centenar de capítulo de libros y varios libros en el ámbito de la Comunicación Política. Ha participado en numerosos proyectos de investigación competitivos, formando parte de tres grupos de investigación en Comunicación, Política e Imagen (UCAM), Ciberdemocracia (UCM), y Comunicación, Cultura y Tecnología (UM). Cuenta con un sexenio de investigación activo y es miembro activo de diferentes asociaciones profesionales internacionales, además de evaluadora de diferentes revistas.

rzamoramedina@um.es

Î́ndice $\mathbf{H}: 10$

Orcid ID: http://orcid.org/0000-0002-0541-2456

Google Scholar: http://scholar.google.es/citations?user=uhqk4acAAAAJ\&hl=en 